\title{
DECADE IN REVIEW-CANCER IMMUNOTHERAPY:
}

\section{Entering the mainstream of cancer treatment}

\section{Steven A. Rosenberg}

National Cancer Institute, 10 Center Drive MSC 1201, Bethesda, MD 20892, USA. sar@nih.gov

\begin{abstract}
Administration of IL-2, the first immunotherapy capable of mediating complete, durable cancer responses was originally described in 1985 and approved by the FDA for the treatment of patients with renal cancer and melanoma in 1992 and 1998, respectively. In 2003, the first evidence that the administration of an antibody targeting the inhibitory T-cell costimulatory molecule CTLA-4, (often referred to as a checkpoint modulator) could mediate cancer regression in patients with metastatic melanoma was published. Despite extensive efforts to develop therapeutic cancer vaccines, prior to 2004 none had been shown to be effective.
\end{abstract}

The first effective adoptive cell-transfer (ACT) immunotherapy for cancer was reported in 1988 and showed that cultured tumour infiltrating lymphocytes (TIL) isolated from resected metastatic melanomas and administered to the autologous patients could mediate objective cancer regressions (Figure 1). The effectiveness of this T-cell therapy was dramatically improved in 2002 with the discovery that lympho depletion prior to T-cell administration could improve the antitumour activity of the transferred cells. Thus by 2004, there were clear indications that either direct stimulation of $\mathrm{T}$ cells, blockade of checkpoint modulators or the administration of antitumour $\mathrm{T}$ cells could mediate regression of invasive, vascularized cancers in humans.

In the subsequent decade, increasing information about the importance of cell-surface inhibitory molecules present on lymphocytes led to striking advances in immunotherapy, and antibodies that could block these inhibitory receptors were administered to patients. The first randomized protocol to demonstrate a survival benefit for a cancer immunotherapy was published by Hodi et al. ${ }^{1}$ in 2010 . In this study, patients with metastatic melanoma were randomly assigned to receive a peptide vaccine alone or in combination with anti-CTLA-4 antibody (ipilimumab) therapy. ${ }^{1}$ This trial was controversial, as it was already known that ipilimumab could mediate objective (and sometimes complete) responses in patients with melanoma, whereas peptide vaccines alone had virtually no evidence of efficacy, and no crossover between cohorts was allowed. In the vaccine plus ipilimumab arm, 35 of 540 patients (6.5\%) experienced an objective response compared with two of 136 patients (1.5\%) treated with peptide alone. ${ }^{1}$ Of note, the patients who received ipilimumab showed an increase in median survival from 6.5 months to 10 months. ${ }^{1}$ These results ultimately led to the approval of ipilimumab for the treatment of patients with metastatic melanoma by the

Competing interests

The author declares no competing interests. 
FDA in March 2011. The clinical value of ipilimumab in other cancer types (with the possible exception of renal cancer) is currently unknown.

Clinical utility of the blockade of a second inhibitory lymphocyte receptor programmed cell death 1 (PD-1) was anticipated in 2010 and firmly demonstrated in 2012 in a successive trial that used this agent in 296 patients with different cancer types. ${ }^{2}$ Among 236 evaluable patients, objective response rates were seen in $18 \%, 28 \%$ and $27 \%$ of patients with nonsmall-cell lung cancer, melanoma and renal cell cancer, respectively. ${ }^{2}$ No objective responses were observed in patients with colorectal or prostate cancers. ${ }^{2}$ Response rates seemed to be increased when PD-L1, the ligand for PD-1, was expressed on the cancer cells, and the use of a blocking antibody against PD-L1 resulted in responses in nine of $52(17 \%)$ patients with melanoma, two of $17(12 \%)$ with renal cancer, five of $49(10 \%)$ with nonsmall-cell lung cancer and one of $17(6 \%)$ patients with ovarian cancer. ${ }^{3}$ The impressive responses seen with pembrolizumab, an anti-PD-1 antibody, in multicentre phase II studies led to approval of this agent by the FDA in September 2014 for the treatment of patients with metastatic melanoma refractory to ipilimumab and to a BRAF inhibitor, if the tumour was BRAF V600-mutation-positive. Evaluation of the blockade of other inhibitory receptors such as TIM-3 or LAG-3 is ongoing.

Very little progress has been made in the past 10 years concerning the application of therapeutic cancer vaccines. The only positive randomized trial of a therapeutic vaccine was published by Kantoff et al. ${ }^{4}$ in July 2010. Patients with hormone-refractory prostate cancer received the administration of autologous peripheral blood mono nuclear cells activated $e x$ vivo with a recombinant protein consisting of prostatic acid phosphatase fused to granulocyte-macrophage colony stimulating factor (sipuleucel-T vaccine) or placebo. ${ }^{4}$ Median survival improved from 21.7 months in the placebo cohort to 25.8 months in the vaccine group. ${ }^{4}$ Such increase in median survival was surprising as only one of the 341 patients $(0.3 \%)$ treated with the vaccine had an objective response, and only $2.6 \%$ of patients had a decrease in serum prostate-specific antigen (PSA) levels $;{ }^{4}$ the time to disease progression was similar in the two groups (14.4 weeks compared to 14.6 weeks) ${ }^{4}$ On the basis of these results, the sipuleucel-T vaccine was approved by the FDA in 2010. So far, this modest clinical result remains the only trial to show a convincing benefit of an active immunization approach to cancer treatment.

"...has moved immunotherapy from the sidelines of cancer treatment into the mainstream of modern oncology"

The past 10 years have seen consider-able progress in the development of ACT immunotherapies using both naturally occurring and genetically engineered lymphocytes. Long-term follow-up studies showing that ACT immunotherapy using autologous TIL could mediate durable complete (and likely curative) responses in patients with refractory metastatic melanoma was published in 2011. ${ }^{5}$ In a series of three sequential trials that used increasing lymphodepleting regimens, objective cancer regressions were seen in 52 of 93 patients (56\%), including 20 patients (22\%) with complete regressions. 19 of the 20 patients had ongoing complete responses beyond 5 years, with the longest ongoing responses persisting beyond 10 years. ${ }^{5}$ These results represented the highest reported response rates of any clinical trials in patients with metastatic melanoma. Furthermore, Bollard et al. ${ }^{6}$ reported 
responses in patients with Epstein-Barr virus (EBV)-related Hodgkin or non-Hodgkin lymphomas receiving autologous cytotoxic $\mathrm{T}$ cells that target the EBV latent membrane proteins LMP2 or LMP1. Specifically, 13 of 21 patients with measurable relapsed or resistant lymphomas exhibited clinical responses, including 11 complete responses. ${ }^{6}$

The difficulty in identifying cells with in vitro antitumour activity against many cancer types led to extensive efforts to genetically engineer T cells for use in ACT therapy. Retroviruses encoding either conventional a $\beta$ T-cell receptors (TCR) or chimeric antigen receptors (CAR; composed of a single chain antibody fused to intracellular signalling chains) are capable of inserting genes into the genome of human lymphocytes with efficiencies exceeding $80 \%$. The first successful use of genetically engineered lymphocytes to mediate cancer regression was reported in 2006. Morgan et al. ${ }^{7}$ showed that retroviral transduction of autologous lymphocytes with the gene encoding a TCR against the melanocyte/melanoma differentiation antigen, MART-1, mediated objective cancer regressions in two of 15 (13\%) patients with metastatic melanoma. Objective cancer regressions were later reported in $30 \%$ of 20 patients receiving gene-modified cells targeting the MART- 1 antigen and in 19\% of 16 patients who received autologous cells transduced with a mouse TCR that recognized the gp100 antigen, demonstrating for the first time that adoptive transfer of genetically modified cells could mediate cancer regression. In 2008, Pule et al. ${ }^{8}$ reported that EBV-specific autologous $\mathrm{T}$ cells transduced with a CAR targeting the GD2 molecule mediated objective tumour regressions in two of eight patients with measurable neuroblastoma.

In 2010, the first report of the successful use of CAR-transduced T cells targeting the CD19 molecule in patients with B-cell lymphomas was reported. ${ }^{9}$ A heavily pre-treated patient with advanced lymphoma exhibited a dramatic response after receiving two cycles of autologous anti-CD19 CAR-transduced T cells and remained progression-free 5 years later. ${ }^{9}$ Additional reports confirmed the ability of these anti-CD19 CAR-transduced T cells to mediate the regression of both indolent as well as aggressive large B-cell lymphomas, and acute and chronic lymphoblastic leukaemias. These early studies using gene-modified cells have greatly expanded the reach of ACT therapy to different tumour types.

The greatest obstacle to the further development of cancer immunotherapy is the identification of target molecules expressed on cancer cells and not on essential human tissues. Immunogenic mutations expressed in individual cancers could be the ideal immunotherapy target. In 2014, Tran et al. ${ }^{10}$ reported a general technique for the identification of lymphocytes capable of targeting the rare but unique immunogenic mutations present in individual epithelial cancers and used this ACT approach to mediate objective regression in a patient with metastatic cholangiocarcinoma. The intrinsic ability of the immune system to specifically target the unique mutations expressed by common cancers is likely to be the key to further progress in the field of cancer immunotherapy.

\section{References}

1. Hodi FS et al. Improved survival with ipilimumab in patients with metastatic melanoma. N. Engl. J. Med 363, 711-723 (2010). [PubMed: 20525992]

2. Topalian SL et al. Safety, activity, and immune correlates of anti-PD-1 antibody in cancer. N. Engl. J. Med 366, 2443-2454 (2012). [PubMed: 22658127] 
3. Brahmer JR et al. Safety and activity of anti-PD-L1 antibody in patients with advanced cancer. N. Engl. J. Med 366, 2455-2465 (2012). [PubMed: 22658128]

4. Kantoff PW et al. Sipuleucel-T immunotherapy for castration-resistant prostate cancer. N. Engl. J. Med 363, 422 (2010).

5. Rosenberg SA et al. Durable complete responses in heavily pretreated patients with metastatic melanoma using T-cell transfer immunotherapy. Clin. Cancer Res 17, 4550-4557 (2011). [PubMed: 21498393]

6. Bollard CM et al. Sustained complete responses in patients with lymphoma receiving autologous cytotoxic T lymphocytes targeting Epstein-Barr virus latent membrane proteins. J. Clin. Oncol 32, 798-808 (2012).

7. Morgan RA et al. Cancer regression in patients after transfer of genetically engineered lymphocytes. Science 314, 126-129 (2013).

8. Pule MA et al. Virus-specific T cells engineered to coexpress tumor-specific receptors: persistence and antitumor activity in individuals with neuroblastoma. Nat. Med 14, 1264-1270 (2008).

[PubMed: 18978797]

9. Kochenderfer JN et al. Eradication of B-lineage cells and regression of lymphoma in a patient treated with autologous T cells genetically engineered to recognize CD19. Blood 116, 4099-4102 (2010). [PubMed: 20668228]

10. Tran E et al. Cancer immunotherapy based on mutation-specific $\mathrm{CD} 4^{+} \mathrm{T}$ cells in a patient with epithelial cancer. Science 344, 641-645 (2014). [PubMed: 24812403] 
By November 2004, when the first issue of Nature Reviews Clinical Oncology was published, cancer immunotherapy had been successfully applied to the treatment of selected human cancers; however, dramatic progress in the following decade has moved immunotherapy from the sidelines of cancer treatment into the mainstream of modern oncology. 


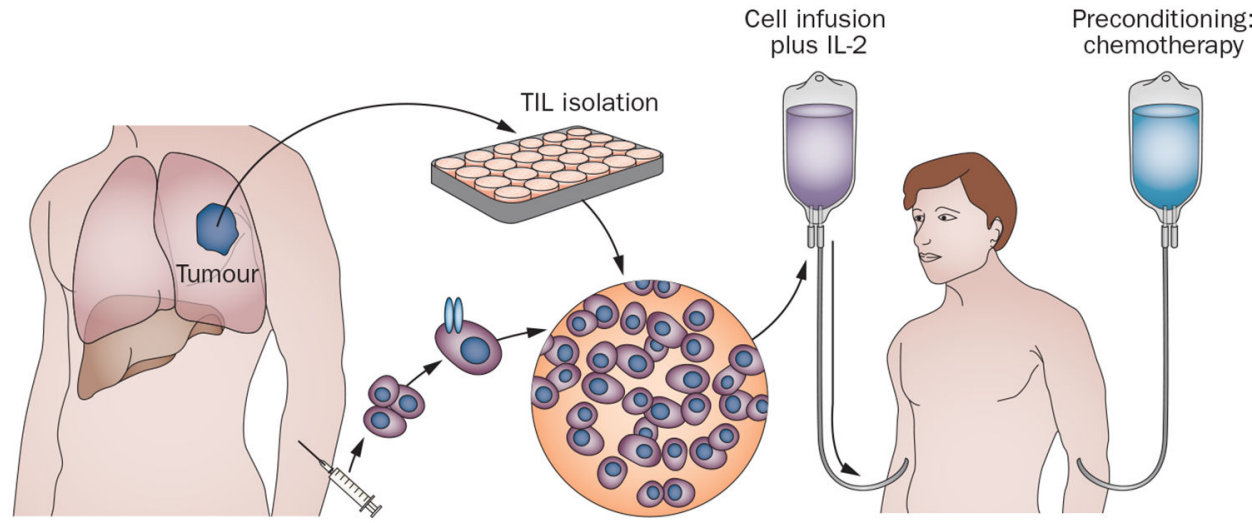

Figure 1 .

Adoptive cell transfer immunotherapy. Cells are obtained from a resected tumour (autologous TILs) or using peripheral lymphocytes genetically engineered to express antitumour T-cell receptors, expanded in vitro and infused to patients after they have received a preparative lymphodepleting regimen. Abbreviation: TIL, tumour-infiltrating lymphocyte. 